\title{
THE COSMIC RAY INCREASES AT 35 AND 60 KYR BP
}

\author{
V Florinski ${ }^{1} \bullet$ W I Axford ${ }^{2} \bullet \mathrm{G} \mathrm{P} \mathrm{Zank}^{3}$ \\ Institute of Geophysics and Planetary Physics, University of California, Riverside, California 92521, USA.
}

\begin{abstract}
Concentrations of ${ }^{10} \mathrm{Be}$ in ice cores and marine sediments exhibit 2 peaks with significant enhancements at 35,000 and 60,000 BP. This radioisotope is produced in the upper atmosphere by spallation of cosmic-ray protons and secondary neutrons on atmospheric nitrogen and oxygen. Previously suggested explanations for the increases include geomagnetic field reversals, a decrease in solar activity, and a supernova explosion. We propose an alternative explanation which involves a change in the galactic environment of the solar system. The structure of the heliosphere is investigated for a period when the Sun enters a cold, dense, unmagnetized interstellar cloud. Under these conditions, the heliosphere contracts to $25 \%$ its present size, significantly affecting galactic cosmic ray modulation and increasing anomalous cosmic ray fluxes. A tenfold increase in anomalous cosmic ray flux and a twofold increase in galactic cosmic ray intensity at Earth are possible in this highdensity case if heliosheath modulation is reduced. We show that this increase in galactic cosmic ray intensity could be responsible for the peaks in ${ }^{10} \mathrm{Be}$ records.
\end{abstract}

\section{INTRODUCTION}

Paleoisotope records from ice cores and deep-sea sediments offer valuable information about the intensity of cosmic rays fluxes at the top of Earth's atmosphere over tens of thousands of years. A well-publicized feature of the ${ }^{10} \mathrm{Be}$ record is 2 prominent peaks at $35,000-40,000$ and 60,000-65,000 BP, first discovered in Antarctic ice cores (Raisbeck et al. 1987). The more recent peak was also detected in ice cores from Greenland (Wagner et al. 2000) and marine sediments (Cini Castagnoli et al. 1995; McHargue et al. 1995; Robinson et al. 1995; Cini Castagnoli et al. 1998). The second peak was also found in sea sediments (McHargue et al. 2000). The duration of the enhanced intensity is of the order of $2 \mathrm{kyr}$.

${ }^{10} \mathrm{Be}$ is a cosmogenic isotope produced in Earth's upper atmosphere by nuclear reactions of cosmicray particles with atmospheric nitrogen and oxygen. Cross-section analysis indicates an energy threshold of $50 \mathrm{MeV}$ for protons. However, the bulk of the production is from secondary neutrons with energies above $10 \mathrm{MeV}$, produced in atmospheric cascades from primary cosmic rays (Masarik and Beer 1999). The rate of production strongly depends on geomagnetic latitude because Earth's magnetic field prevents lower energy charged particles from reaching the atmosphere.

The global occurrence of the peaks points to the increased production rate and, therefore, an enhanced cosmic-ray flux at the top of the atmosphere, as the cause. Several hypotheses have been suggested to explain the increases in production. The most popular current explanation is that they are caused by geomagnetic field excursions, or incomplete reversals (McHargue et al. 2000; Wagner et al. 2000), when the field intensity was only $10-20 \%$ of its present value. The $35 \mathrm{kyr}$ peak (possibly a double peak) appears to correlate with the Mono Lake and Laschamps geomagnetic excursions (Laj et al. 2002). However, the results of Cini Castagnoli et al. (1998) appear to contradict the geomagnetic hypothesis because ${ }^{10} \mathrm{Be}$ in Mediterranean sea sediments starts to increase about $2000 \mathrm{yr}$ before the drop in magnetization measured in the same core sample.

The geomagnetic hypothesis largely relies on the assumption that ${ }^{10} \mathrm{Be}$ is distributed evenly across the globe rather than being precipitated locally, i.e., at the latitude it was produced. Latitudinal mix-

${ }^{1}$ Corresponding author. Email: vflorins@citrus.ucr.edu.

${ }^{2}$ Email: ian@axford.org.

3Email: zank@ucrac1.ucr.edu. 
ing in the stratosphere, where the isotope spends about a year on average before being deposited, determines whether or not polar ${ }^{10} \mathrm{Be}$ will be sensitive to geomagnetic variations (Beer 2000). Measurements performed in the Northern (Brown et al. 1989) and Southern (Graham et al. 2003) Hemispheres at similar latitudes $\left(\sim 40^{\circ}\right)$ but different longitudes show similar deposition rates, which could imply that mixing is taking place at mid-latitudes. Still, there is no consensus as to whether ${ }^{10} \mathrm{Be}$ deposited near the poles is derived from lower latitudes, and supporting experimental evidence both for (Beer et al. 1990) and to the contrary (Steig et al. 1996) is available.

Several alternative hypotheses have been proposed to explain the peaks. A prolonged decrease in solar activity (i.e. a "Maunder Minimum" lasting 2 millennia) could have caused a reduction in the heliospheric modulation of galactic cosmic rays (GCR). This scenario is considered unlikely in view of the absence of any evidence for such extended periods of negligible solar activity (see Sonett et al. 1987). Another hypothesis involves the interaction of a supernova blast wave with the solar system, carrying with it an increase in shock-accelerated protons (Sonett et al. 1987; Kocharov 1994). The Geminga pulsar at a distance of $160 \mathrm{pc}$ has been suggested as a remnant of the supernova that caused the anomalies (Ramadurai 1995; Ellis et al. 1996). Axford (1981) estimated that the upper limit on the rate of supernova shock passage of any given point in space is only about $4 \times 10^{-7} \mathrm{yr}^{-1}$ for shocks that are capable of producing a twofold increase in GCR pressure. The actual rate will be smaller owing to shock deceleration by the cosmic-ray pressure. It is also worth noting that the concept of a recent nearby supernova explosion contradicts the theory of the origin of the "Local Bubble," where the latter is produced by a sequence of supernova explosions at a much earlier time (Smith and Cox 2001).

A further hypothesis was put forward by Zank and Frisch (1999). They suggested that the solar system may have passed through a relatively small $(0.05$ parsec), dense molecular cloud, causing the heliosphere to shrink. Binary star system observations show that dense clouds possess structure on scales at and below $0.05 \mathrm{pc}$, although the data available is not yet sufficient to construct a distribution of these small clouds (Lauroesch and Meyer 1999). Zank and Frisch (1999) estimated that the Sun may enter a region with a higher hydrogen density in $10^{4}$ to $10^{6} \mathrm{yr}$. They also argued that such a condition would result in a decrease of GCR modulation and an increase in anomalous cosmic ray (ACR) flux at Earth. Nevertheless, it remains to be seen to what extent this change in the solar environment could affect ${ }^{10} \mathrm{Be}$ production rates. Here, we calculate the global structure of the heliosphere, including the location of the 3 major discontinuities and the pickup ion intensities, and use this data to estimate the change in cosmic-ray intensities at Earth.

\section{DESCRIPTIVE BACKGROUND}

As the solar wind collides with the interstellar plasma flowing past the solar system, an interface is formed consisting of 2 shocks and a contact discontinuity, the heliopause, separating the plasmas. The region inside the heliopause, the heliosphere, is filled with a highly irregular and turbulent magnetic field of solar origin, providing an obstacle for galactic cosmic ray propagation. The process (modulation) is a combination of drift, diffusion, and cooling, or acceleration of the energetic particles in the solar wind region inside the termination shock (TS), and in the heliosheath between the TS and the heliopause. As shown in Florinski et al. (2003), at least half of the total amount of GCR modulation occurs in the heliosheath. Present heliospheric models place the TS at about 90-100 AU from the Sun and the heliopause at about $150 \mathrm{AU}$ in the upwind direction, based on the observed properties of the local interstellar medium (Frisch 2000). The termination shock is also a source of anomalous cosmic rays which are accelerated to tens of $\mathrm{MeV}$ per nucleon from a pool of pre-energized pickup ions (see Fichtner 2001 for a review). 
In order to estimate GCR modulation in the high-density cloud, we must self-consistently calculate the structure of the heliosphere. We assume the interstellar cloud to have a number density of $8.5 \mathrm{~cm}^{-3}$, which is 40 times that of the total number density of the Local Cloud presently surrounding the solar system (Frish 2000; Lallement 2001). Because of its high density, the cloud is expected to be cold, with a temperature of only $200 \mathrm{~K}$, in order to be in pressure equilibrium with the surrounding warm interstellar medium. This implies that the cloud consists primarily of neutral hydrogen atoms and molecules, i.e., its degree of ionization is close to zero. We chose the relative velocity between the cloud and the solar system to be equal to the Local Cloud velocity of $25 \mathrm{~km} / \mathrm{s}$.

Interstellar atoms have a profound effect on the structure of the heliosphere (see Zank 1999 for a review). Atoms can exchange electrons with solar wind ions, producing a new population of ions (pickup ions) and atoms. Because interstellar atoms tend to be less energetic than the solar wind ions, the solar wind loses energy and decelerates. This results in a significant (30-40\%) reduction of the distances to the heliospheric boundaries. In the high-density cloud with the LISM neutral hydrogen density some 50 times higher than at present and with no ionized LISM component, one could expect that the solar wind would be drastically decelerated by charge-exchange momentum removal and not undergo a termination shock transition. However, as we show below, pickup ions produced as a result of charge exchange and photoionization provide the missing confining pressure, ensuring that a shock transition still occurs. Moreover, neutral filtration in the outer heliosphere, similar to that predicted by heliospheric models (Baranov and Malama 1993; Pauls et al. 1995; Zank et al. 1996; Fahr et al. 2000), is expected to reduce the density of neutral hydrogen atoms reaching the inner heliosphere.

We use a two-fluid (plasma + neutral) model to describe the flow of partially ionized gas in and around the heliosphere. The present model is based on the work of Florinski et al. (2003) and includes charge exchange and photoionization processes. To estimate the effect of the changed size of the heliosphere on GCR modulation and ACR production, we use a simple one-dimensional model and solve the Parker transport equation for the phase space density $f(r, p)$ using spherical symmetry ( $p$ is the particle momentum). We emphasize that the simple spherical model is used only to explore cosmic-ray intensities at $1 \mathrm{AU}$ in a heliosphere that is much smaller in size than at present, rather than to perform a detailed calculation of cosmic-ray density at all heliocentric distances and latitudes. To accomplish the latter, a more detailed model, including the magnetic field geometry and drift effects, should be used (e.g. Jokipii et al. 1993; Steenberg and Moraal 1996).

\section{RESULTS AND DISCUSSION}

Figure 1 shows the number density and plasma streamlines in the heliosphere submersed in a highdensity interstellar cloud. This result was obtained with a two-fluid plasma-neutral model, taking into account charge exchange and photoionization (Florinski et al. 2003). A similar calculation was performed by Scherer et al. (2002) for a partially ionized cloud with a density 3 times smaller. Our results differ in a significant way from those of Scherer et al. (2002) owing to a difference in our treatment of cosmic-ray propagation.

The heliospheric interface in Figure 1 contains 2 shocks and the heliopause, visible as a surface separating the solar and the interstellar streams. The TS inside the heliopause is greatly elongated in the tailward direction. The heliopause is located at a distance of only $31 \mathrm{AU}$ in the upwind direction, while the bow shock (visible outside the heliopause) is located at $40 \mathrm{AU}$ upstream. Inside the TS, the number density of $H$ atoms decreases monotonically with decreasing heliocentric distance. The neutral hydrogen density inside the TS may be fitted by a power law, such that $n_{H}=n_{H 0}\left(r / r_{0}\right)^{b}$ with 


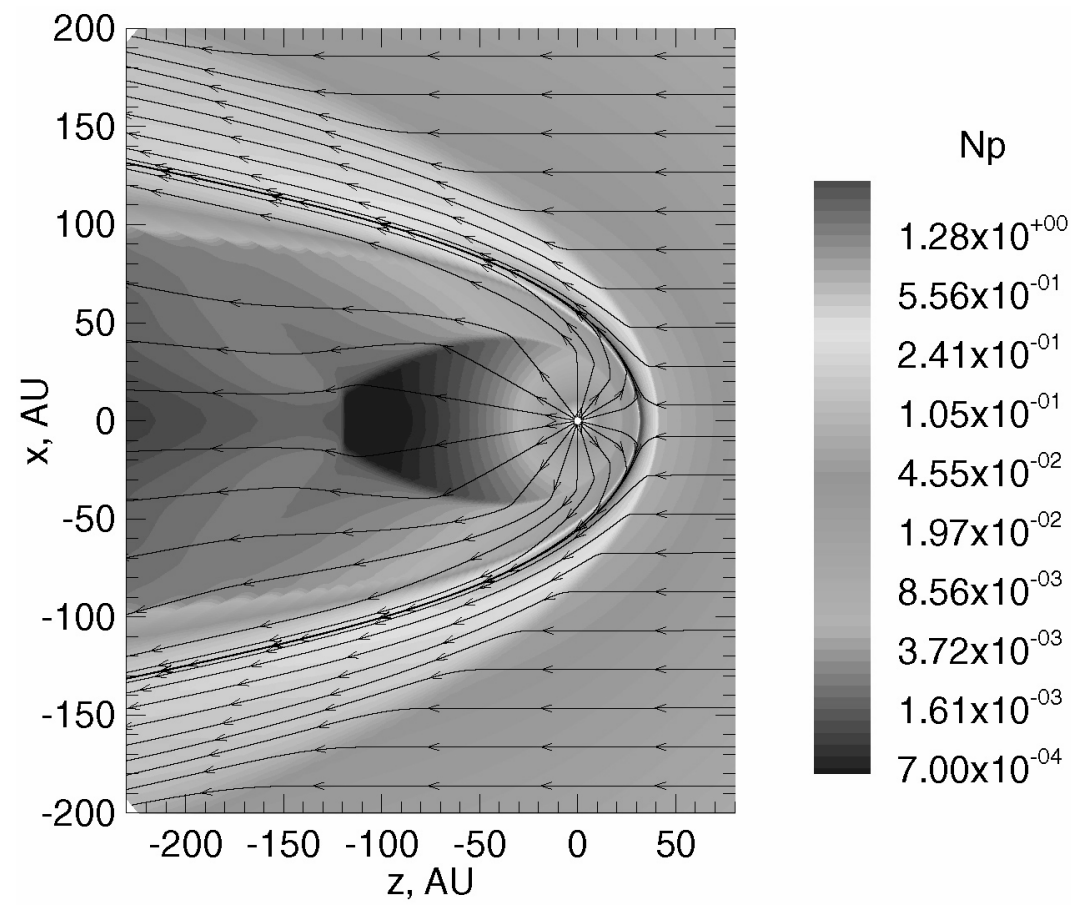

Figure 1 Plasma density (logarithmic scale) and streamlines showing the heliospheric interface in a dense interstellar cloud.

$n_{H 0}=3 \times 10^{-3} \mathrm{~cm}^{-3}$ and $b=1.7$. Such a rapid density decrease is caused by a strong deflection of $\mathrm{H}$ atoms by the hydrogen wall interaction region between the heliopause and the bow shock, effectively excluding the neutrals from the inner heliosphere. The termination shock is located at $23 \mathrm{AU}$ upwind, $33 \mathrm{AU}$ in the crosswind direction, and at $120 \mathrm{AU}$ in the tail. The solar wind is decelerated from $508 \mathrm{~km} / \mathrm{s}$ at the inner boundary ( $1 \mathrm{AU}$ ) to $400 \mathrm{~km} / \mathrm{s}$ just before the TS along the symmetry axis. Our results differ from previous work of Zank and Frisch (1999) in that we did not obtain a highly unsteady solution shown in their work. We also do not observe the twofold decrease in the wind speed as suggested by Scherer et al. (2002). Note that the termination shock in our model is relatively weak as a result of pickup ion heating, with a compression ratio of 3.1 in the upwind direction.

The Parker transport equation for the cosmic-ray phase space density $f(r, p)$ is solved numerically for a radial diffusion coefficient of the form

$$
\kappa_{r r}=\kappa_{0} \frac{w}{c}\left(\frac{p}{p_{0}}\right)^{\alpha} \frac{r}{r_{0}}\left[1+\left(\frac{r_{1}}{r}-1\right) H\left(r-r_{1}\right)\right]\left[1-(1-s) H\left(r-r_{s}\right)\right]
$$

where $p_{0}$ corresponds to $1 \mathrm{GV}$ rigidity, $r_{0}=1 \mathrm{AU}, w$ is the particle speed, $r_{s}$ is the distance to the shock, and $H$ is the Heavyside step function. The radial dependence implies that diffusion increases linearly as a function of $r$ at small heliocentric distances but becomes independent of $r$ beyond $r_{1}$. Such a radial dependence may be expected if pickup ion-driven turbulence is present at sufficient levels to compensate for the expansion (Zank et al. 1998). We use $\kappa_{0}=2.2 \times 10^{22} \mathrm{~cm}^{2} s^{-1}, \alpha=1.1$, $r_{1}=19 \mathrm{AU}$, and $s=0.14$ for the present heliosphere. For the high-density case, we reduce the size of the simulation domain by a factor of 4 . The small diffusion downstream of the shock simulates the effect of the "modulation wall" bordering the heliopause (Florinski et al. 2003). When used in a 
spherical model, the parameters above allow for modeling of the observed ACR (Cummings et al. 2002) and GCR (McDonald 1998) solar minimum fluxes with a reasonable degree of accuracy.

Because of a higher neutral density inside the TS, we would expect elevated turbulence levels and, hence, a reduced diffusion coefficient in the inner heliosphere. To estimate this reduction, we use an incompressible spherically-symmetric MHD turbulence model of Zank et al. (1998) to calculate turbulence generation by pickup ion instabilities. The pickup ion number density $n_{\mathrm{i}}$ may be found from the expression for $n_{H}$ given above as

$$
n_{i}=\frac{n_{H 0} n_{p 0} \sigma r_{0}^{2-b} r^{b-1}}{b+1}
$$

where $n_{p 0}=5 \mathrm{~cm}^{-3}$ is the solar wind density at $1 \mathrm{AU}$ and $\sigma=2.4 \times 10^{-15} \mathrm{~cm}^{2}$ is the cross-section for charge exchange. Our calculations of the turbulent energy density show that it is a factor of 2 to 4 greater in the high-density case than at present. To model the effect of reduced diffusion, we decrease the value of $r_{l}$ in Equation 1 to $5 \mathrm{AU}$ for anomalous cosmic rays. However, we leave the diffusion coefficient for the galactic cosmic rays unchanged because this highly energetic population is not likely to be strongly affected by small-scale PUI turbulence. Equation 2 yields $n_{i}=1.8 \times$ $10^{-3} \mathrm{~cm}^{-3}$ at the TS, which is approximately 20 times that calculated by Fahr et al. (2000) for the present heliosphere. Because the production rate of ACRs is directly proportional to the rate of injection of PUI into the diffusive shock acceleration process, which, in turn, is expected to be proportional to the PUI fluxes through the shock, we increase the injection rate by the same factor of 20 .

Figure 2a compares GCR intensities for a high-density cloud and present-day conditions, while Figure $2 \mathrm{~b}$ shows ACR intensities. The left panel of Figure $2 \mathrm{a}$ refers to the present heliosphere with a termination shock at $90 \mathrm{AU}$ and the external boundary at $150 \mathrm{AU}$, while the right panel shows intensities for a high-density case with a shock at $23 \mathrm{AU}$ and the boundary at $31 \mathrm{AU}$. This plot demonstrates an intensity enhancement by a factor of $1.5-4$ between $100 \mathrm{MeV}$ and $1 \mathrm{GeV}$ (particles which are likely to have the greatest impact on ${ }^{10} \mathrm{Be}$ production) compared with the present heliosphere. This increase is mostly a consequence of the reduced thickness of the "modulation wall," which can be seen by comparing spectra at the termination shock for the 2 cases. Increased GCR intensity implies that ${ }^{10} \mathrm{Be}$ production rates would also increase by the same amount, which is sufficient to explain the peaks. Note that Scherer et al. (2002) calculated a similar GCR enhancement, but without including heliosheath modulation. In their case, the increase was caused by a reduction of the size of the modulation region under the assumption that the diffusion coefficient was nearly independent of radial distance, which is difficult to justify in view of a relatively rapid ( $\sim r$ or faster) increase in the particle gyroradius with heliocentric distance.

Anomalous cosmic ray intensities are shown in Figure 2b. In the left panel, corresponding to the present heliosphere, the shock compression ratio was taken to be 3.5 (Florinski et al. 2003).The spectrum shown in the right panel is steeper at lower energy because of the reduced shock compression ratio. The $1 \mathrm{AU}$ intensity at $100 \mathrm{MeV}$ is increased by a factor of 10 . Nevertheless, it remains below the GCR intensity shown in the right panel of Figure 2a, especially at higher energies, implying that ACR will have only a small contribution to ${ }^{10} \mathrm{Be}$ production. This result formally agrees with that of Scherer et al. (2002). However, these authors used the same ACR spectra at the TS and diffusion coefficients to obtain both high- and low-density results, in contrast to our analysis. 

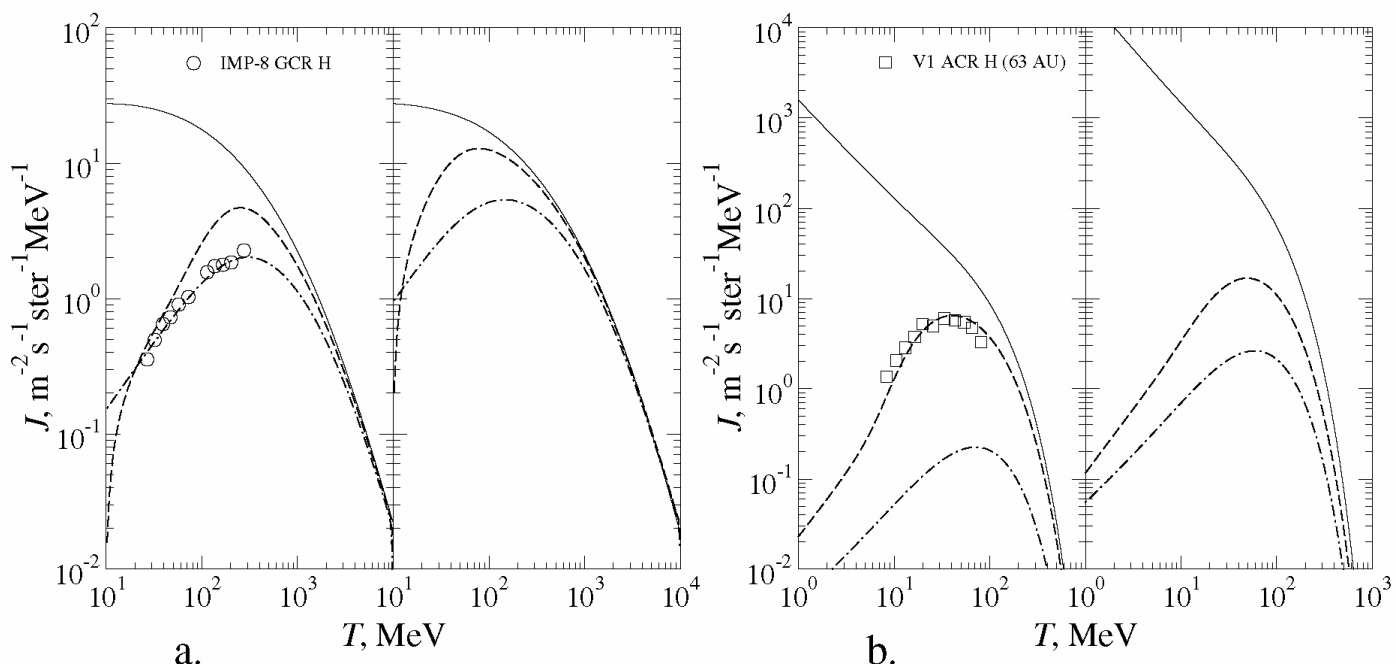

Figure 2 (a) Left panel: present-day GCR intensities at the heliopause (solid line), termination shock (dashed) and Earth (dash-dotted). Right panel: same for the high-density case with the shock at $23 \mathrm{AU}$ and the heliopause at $31 \mathrm{AU}$. Circles show IMP-8 data from McDonald (1998); (b) Left panel: current anomalous hydrogen intensities at the TS (solid line), 63 AU (dashed) and Earth (dash-dotted). Right panel: same for the high-density case. The dashed line is the intensity at $10 \mathrm{AU}$. Squares show Voyager 1 data from Cummings et al. (2002).

\section{CONCLUSION}

We have examined the possibility of enhancements in cosmic-ray proton fluxes during Earth's history caused by the passage of the solar system through relatively small molecular clouds with a density some 40 times higher than the LISM today. We found that the size of the heliosphere is reduced by a factor of about 4-5 compared to its present state, and that the neutral hydrogen density is increased by a factor of 7 at the termination shock located at $23 \mathrm{AU}$. Our calculations of anomalous cosmic-ray distribution show a tenfold intensity increase compared to the present heliosphere. Nevertheless, these particles are not very likely to be present in sufficiently high numbers at above the threshold energy for ${ }^{10} \mathrm{Be}$ production. Due to a limitation of our model, we did not consider the possibility of a strongly modified turbulent shock, which requires further investigation. We also found that galactic cosmic ray intensities may be enhanced by a factor of 1.5 to 4 between $100 \mathrm{MeV}$ and $1 \mathrm{GeV}$. This conclusion depends critically on the amount of modulation inside the "modulation wall" region. The calculated increase in cosmic-ray fluxes at Earth under the conditions inside a dense interstellar cloud is sufficient to explain the twofold increases in ${ }^{10} \mathrm{Be}$ production rate in the past.

\section{ACKNOWLEDGEMENTS}

V Florinski and G P Zank acknowledge the support by NSF grant ATM-0296114 and NASA grants NAG5-11621 and NAG5-12903. W I Axford was supported by a Regents Professor appointment at the UC Riverside. Numerical computations were performed on the IGPP/UCR "Lupin" cluster. 


\section{REFERENCES}

Axford WI. 1981. Acceleration of cosmic rays by shock waves. Proceedings of the 17th International Cosmic Ray Conference (Paris) 12:155-204.

Baranov VB, Malama YG. 1993. Model of the solar wind interaction with the local interstellar medium: numerical solution of self-consistent problem. Journal of Geophysical Research 98:15,157-63.

Beer J. 2000. Long-term indirect indices of solar variability. Space Science Reviews 94:53-66.

Beer J, Blinov A, Bonani G, Finkel RC, Hofmann HJ, Lehmann B, Oeschger H, Sigg A, Schwander J, Staffelback T, Stauffer B, Suter M, Wölfli W. 1990. Use of ${ }^{10} \mathrm{Be}$ in polar ice to trace the 11-year cycle of solar activity. Nature 347:164-6.

Brown L, Stensland GJ, Klein J, Middleton R. 1989. Atmospheric deposition of ${ }^{7} \mathrm{Be}$ and ${ }^{10} \mathrm{Be}$. Geochimica et Cosmochimica Acta 53:135-42.

Cini Castagnoli G, Albrecht A, Beer J, Bonino G, Shen C, Callegari E, Taricco C, Dittrich-Hannen B, Kubik P, Suter M, Zhu GM. 1995. Evidence for enhanced ${ }^{10} \mathrm{Be}$ deposition in Mediterranean sediments 35 kyr BP. Geophysical Research Letters 22:707-10.

Cini Castagnoli G, Bonino G, Taricco C, Lehman B. 1998. Cosmogenic isotopes and geomagnetic signals in a Mediterranean sea sediment at 35,000 yr BP. Il Nuovo Cimento 21:243-6.

Cummings AC, Stone EC, Steenberg CD. 2002. Composition of anomalous cosmic rays and other heliospheric ions. Astrophysical Journal 578:194-210.

Ellis J, Fields BD, Schramm DN. 1996. Geological isotope anomalies as signatures of nearby supernovae. Astrophysical Journal 470:1227-36.

Fahr HJ, Kausch T, Scherer H. 2000. A 5-fluid hydrodynamic approach to model the solar system-interstellar medium interaction. Astronomy and Astrophysics $357: 268-82$

Fichtner H. 2001. Anomalous cosmic rays: messengers from the outer heliosphere. Space Science Reviews 95: 639-754.

Florinski V, Zank GP, Pogorelov NV. 2003. Galactic cosmic ray transport in the global heliosphere. Journal of Geophysical Research 108:1228.

Frisch PC. 2000. The galactic environment of the Sun. Journal of Geophysical Research 105:10,279-89.

Graham I, Ditchburn R, Barry B. 2003. Atmospheric deposition of ${ }^{7} \mathrm{Be}$ and ${ }^{10} \mathrm{Be}$ in New Zealand rain (1996-98). Geochimica et Cosmochimica Acta 67: 361-73.

Jokipii JR, Kota J, Merenyi E. 1993. The gradient of galactic cosmic rays at the solar-wind termination shock. Astrophysical Journal 405:782-6.

Kocharov GE. 1994. On the origin of cosmic rays. Astrophysical Letters and Communications 29:227-32.

Laj C, Kissel C, Scao V, Beer J, Thomas DM, Guillou H, Muscheler R, Wagner G. 2002. Geomagnetic intensity and inclination variations at Hawaii for the past $98 \mathrm{kyr}$ from core SOH-4 (Big Island): a new study and a comparison with existing contemporary data. Physics of the Earth and Planetary Interiors 129:205-43.

Lallement R. 2001. Heliopause and asteropauses. Astrophysics and Space Science 277:205-17.

Lauroesch JT, Meyer DM. 1999. Observations of smallscale interstellar structure in dense atomic gas. Astrophysical Journal 519:L181-L184.

Masarik J, Beer J. 1999. Simulation of particle fluxes and cosmogenic nuclide production in the earth's atmosphere. Journal of Geophysical Research 104:12,099111.

McDonald FB. 1998. Cosmic-ray modulation in the heliosphere - a phenomenological study. Space Science Reviews 83:33-50.

McHargue LR, Damon PE, Donahue DJ. 1995. Enhanced cosmic-ray production of ${ }^{10} \mathrm{Be}$ coincident with Mono Lake and Laschamp geomagnetic excursions. Geophysical Research Letters 22:659-722.

McHargue LR, Donahue DJ, Damon PE, Sonett CP, Biddulph D, Burr G. 2000. Geomagnetic modulation of the late Pleistocene cosmic-ray flux as determined by ${ }^{10} \mathrm{Be}$ from Blake Outer Ridge marine sediments. Nuclear Instruments and Methods in Physics Research Section B 172:555-61.

Pauls HL, Zank GP, Williams LL. 1995. Interaction of the solar wind with the local interstellar medium. Journal of Geophysical Research 100:21,595-604.

Raisbeck GM, Yiou F, Bourles D, Lorius C, Jouzel J, Barkov NI. 1987. Evidence for two intervals of enhanced ${ }^{10} \mathrm{Be}$ deposition in Antarctic ice during the last glacial period. Nature 326:273-7.

Ramadurai S. 1995. Very long time variations of the cosmic ray intensity and their origin. Advances in Space Research 15(1):41-8.

Robinson C, Raisbeck GM, Yiou F, Lehman B, Laj C. 1995. The relationship between ${ }^{10} \mathrm{Be}$ and geomagnetic field strength records in central North Atlantic sediments during the last $80 \mathrm{ka}$. Earth and Planetary Science Letters 136:551-7.

Scherer K, Fichtner H, Stawicki O. 2002. Shielded by the wind: the influence of the interstellar medium on the environment of Earth. Journal of Atmospheric and Solar-Terrestrial Physics 64:795-804.

Smith RK, Cox DP. 2001. Multiple supernova remnant models of the Local Bubble and the soft X-ray background. Astrophysical Journal Supplement Series 134:283-309.

Sonett CP, Morfill GE, Jokipii JR. 1987. Interstellar shock waves and ${ }^{10} \mathrm{Be}$ from ice cores. Nature 330 : 458-60.

Steenberg CD, Moraal H. 1996. An acceleration/modulation model for anomalous cosmic ray hydrogen in the heliosphere. Astrophysical Journal 463:776-83.

Steig EJ, Polissar PJ, Stuiver M, Grootes PM, Finkel RC. 1996. Large amplitude solar modulation cycles of 
${ }^{10} \mathrm{Be}$ in Antarctica: implications for atmospheric mixing processes and interpretation of the ice core record. Geophysical Research Letters 23:523-6.

Wagner G, Masarik J, Beer J, Baumgartner S, Imboden D, Kubik PW, Synal H-A, Suter M. 2000. Reconstruction of the geomagnetic field between 20 and $60 \mathrm{kyr}$ BP from cosmogenic radionuclides in the GRIP ice core. Nuclear Instruments and Methods in Physics Research B 172:597-604.

Zank GP. 1999. Interaction of the solar wind with the local interstellar medium: a theoretical perspective. Space Science Reviews 89:413-688.
Zank GP, Frisch PC. 1999. Consequences of a change in the galactic environment of the Sun. Astrophysical Journal 518:965-73.

Zank GP, Matthaeus WH, Bieber JW, Moraal H. 1998. The radial and latitudinal dependence of the cosmic ray diffusion in the heliosphere. Journal of Geophysical Research 103:2085-97.

Zank GP, Pauls HL, Williams LL, Hall DT. 1996. Interaction of the solar wind with the local interstellar medium: a multifluid approach. Journal of Geophysical Research 101:21,639-55. 